\title{
Water Assisted and Acid Catalyzed Decarboxylation Reactions of Ketoprofen in Aqueous Solutions
}

\author{
David Lee Phillips ${ }^{\mathrm{a}, *}$, Ming-De Li ${ }^{\mathrm{a}}$, Yong $\mathrm{Du}^{\mathrm{b}}$ and Jiadan Xue ${ }^{\mathrm{c}}$ \\ ${ }^{a}$ Department of Chemistry, The University of Hong Kong, Pokfulam Road, Hong Kong, P. R. China \\ ${ }^{b}$ Department of Chemistry, The University of Rochester, Rochester, New York, 14627 \\ ${ }^{c}$ Department of Chemistry, The Ohio State University, Columbus, Ohio, 43210
}

Ketoprofen (KP) is a nonsterioidal anti-inflammatory drug (NSAID) that is typically utilized to treat pain and inflammation symptoms. A drawback of the use of $\mathrm{KP}$ is that it can result in photosensitivity and phototoxicity due to its specific structure and electronic characteristics. ${ }^{1}$ A number of studies have been done in recent years to investigate the phototoxic and photoallergic reactions caused by KP. This work wad done in part to estimate the safety of KP and also to better understand the mechanism(s) of the photosensitivity reactions when KP is excited by ultraviolet light. ${ }^{2-5}$ The results from these studies found that the photochemistry of KP in aqueous solution is correlated to its acid-base chemistry. The neutral form of ketoprofen that is present in the absence of a base shows typical benzophenone-like photochemistry ${ }^{6}$, while the deprotonated ketoprofen that is present in the presence of a base undergoes an efficient photodecarboxylation process. ${ }^{6,7}$ The preceding studies indicate the photochemistry of KP is propably determined by the precursors, the neutral form of ${ }^{3}[\mathrm{KP}]$ or the deprotonated ${ }^{3}\left[\mathrm{KP}^{-1}\right] .{ }^{6}$ The character of the carbanion's precursor in the photochemistry of KP was investigated by utilizing transient absorption spectroscopy and quenching experiments in organic solvent-water mixtures. ${ }^{2,8}$ However, it was difficult to clearly tell the different intermediates apart from one another since they have similar electronic absorption spectra and time-scales in the time-resolved transient absorption experiments that gave rise to spectral overlap of the absorption spectra on similar time scales for the different transient species that could be present in the reactions. In these types of reactions, time-resolved vibrational spectroscopy techniques like time-resolved resonance Raman (TR3) spectroscopy are particularly useful to apply since they can better tell the different transient species apart from each other by their vibrational frequencies that act like a fingerprint for each species.

In this presentation, nanosecond time-resolved resonance Raman (TR3) spectroscopy was utilized to investigate the photochemistry of ketoprofen in neutral aqueous solutions with varying water concentrations and also in different acidic solutions with varying $\mathrm{pH}$ values and kinds of acids. ${ }^{9,10}$ The rate constants and reaction mechanism of ketoprofen were found to be greatly dependent on the both the concentration of water and acid in the reaction system. For example, in neat acetonitrile and solvents with low concentrations of water (such as water:acetonitrile $\leq 1: 1, \quad \mathrm{v}: \mathrm{v}), \quad$ ketoprofen shows mostly benzophenone-like 
photochemistry to generate a triplet state that subsequently produces a ketyl radicallike species by a hydrogen abstraction reaction. In contrast to this, in reaction solutions with very high concentrations of water (such as water:acetonitrile $\geq 9: 1, \mathrm{v}: \mathrm{v}$ ), the triplet state ketoprofen is first detected on the nanosecond time scale and then exhibits a very fast decarboxylation reaction to make a triplet protonated biradical carbanion species. For reaction solutions with medium water concentrations (such as between $50 \%$ and $90 \%$ water by volume), the hydrogen abstraction and decarboxylation reactions are two competitive processes with different rate constants which results in the triplet state of ketoprofen to simultaneously produce a ketyl radical species and a triplet protonated biradical carbanion species with the relative population of each dependent on the water concentration. Adding an acid to the aqueous solution speeds up the very fast decarboxylation reaction to make a triplet protonated biradical carbanion species. This acid catalysis was noticeably influenced by the character of the anion of the strong acid used. Density functional theory (DFT) calculations were done to investigate how explicit hydrogen bonding effects of the water molecules influence how the triplet state ketoprofen undergoes a very fast decarboxylation reaction to produce a triplet protonated biradical carbanion species. The results of these DFT calculations were consistent with the TR3 experimental results. Additional DFT calculations were performed with explicit consideration of the acid and its anion in the reaction system and it was found that the binding pattern of the acid and its anion in the water network appears to account for the variations in the kinetics of the decarboxylation with the type of acid used in the reaction system.

\section{ACKNOWLEDGMENTS}

This work was supported by a grant from the Research Grants Council of Hong Kong (HKU 7035/08P), the award of a Croucher Foundation Senior Research Fellowship (2006-07) from the Croucher Foundation and an Outstanding Researcher Award (2006) from the University of Hong Kong to DLP.

\section{REFERENCES}

1 H. Bagheri, V. Lhiaubet, J. L. Montastruc, N. Louis-Lalanne, Drug Safety, 22, 339-349(2000).

2 G. Cosa, L. J. Martinez, J. C. Scaiano, Phys. Chem. Chem. Phys., 1, 3533-3537(1999).

3 S. Monti, S. Sortino, G. D. Guidi, G. Marconi, J. Chem. Soc., Faraday Trans., 93, 22692274(1997).

4 V. Lhiaubet-Vallet, N. Belmadoui, M. J. Climent, M. A. Miranda, J. Phys. Chem. B, 111, 82778282(2007).

5 K. A. K. Musa, J. M. Matxain, L. A. Eriksson, J. Med. Chem., 50, 1735-1743(2007).

6 Y. P. Chuang, J. Xue, Y. Du, M. D. Li, H. Y. An, D. L. Phillips, J. Phys. Chem. B, 113, 1053010539(2009).

7 L. J. Martinez, J. C. Scaiano, J. Am. Chem. Soc., 119, 11066-11070(1997).

8 G. Cosa, M. Lukeman, J. C. Scaiano, Acc. Chem. Res., 42, 599-607(2009).

9 M. D. Li, Y. Du, Y. P. Chuang, J. Xue, D. L. Phillips, Phys. Chem. Chem. Phys., Dol:10. 1039/b919330h(2010)

10 M. D. Li, Y. Du, Y. P. Chuang, J. Xue, D. L. Phillips, manuscript in preparation. 